\title{
Improvement Geometric Parameter Saws And Increasing His(Its) Capacity To Work
}

\author{
SH. IMOMKULOV, Z. ABDUKAHHAROV \\ Senior Lecturer of the Department "General Technical Disciplines", \\ Namangan engineering and technology institute, Namangan city, Uzbekistan, \\ shuhrat19801221@gmail.com. \\ Associate professor of the department "General technical disciplines", \\ Namangan engineering and technology institute, Namangan city, Uzbekistan. \\ zohidjon55@mail.ru.
}

\begin{abstract}
Currently, there is no scientific justification for improving the geometric parameters of the chain saw and the accuracy standards of functional parameters. The issue of improving the geometric parameters of the saw is important and, in this regard, requires urgent resolution. The article deals with issues related to changing the strength of the operating working surfaces of the teeth of a chain saw depending on the design.
\end{abstract}

Keyword - The worker surface, saw, stability, toughness, improvement, stringy mass, qualitative factors, vice formation, pat cleansing production, preparing products, wear capability.

\section{INTRODUCTION}

Currently, on the world market, cotton fiber is the main natural raw material for the production of textile products. According to the international Advisory Committee on cotton (ICAC), China, the United States, India, Pakistan, Brazil and Uzbekistan hold a stable leading position in the supply of cotton fiber to the foreign market. Dynamic and stable development of the cotton gin industry, introduction of modern technological equipment at the enterprises of the industry, increase of efficiency and rational use of production capacities are the basis for the production of competitive products and sales on the world market.

In the world practice, a large amount of research is carried out aimed at the development of innovative equipment and technology, which provides for the effective use of modern science and technology, the modernization of existing equipment and their introduction into production. In this area, the development and improvement of a complex of technical means for manufacturing the main parts of the working bodies of cotton processing machines with the highest efficiency are of particular importance [1].

In our Republic, comprehensive measures are being taken to develop the cotton industry, re-equip and modernize cotton gins, increase the profitability of production and processing of raw cotton, and ensure the competitiveness of products. The Strategy for the development of the Republic of Uzbekistan for 2017-2021 defines tasks, in particular, to "increase the competitiveness of the national economy, reduce energy and resource expenditures, and widely introduce energy-saving technologies". One of these tasks is to create and improve technical means for the rational production of saw blades for cotton processing machines [2].

The modern stage of scientific and technical progress is characterized by rapid improvement of technical parameters of products, intensification of work processes, increasing reliability and resource. Every five to seven years, new generations of machines are created, reflecting the achievements of scientific and technological progress. There is a rapid change of structural materials, new technological processes are introduced. This document is a template. An electronic copy can be downloaded from the conference website. For questions on paper guidelines, please contact the conference publications committee as indicated on the conference website. Information about final paper submission is available from the conference website.

\section{EXPERIMENTAL PROCEDURES.}

Currently, the task is not only to develop the design and technological process, but also to improve their parameters, including the development of the profile saw design [3].

In modern technology, the importance of strength problems increases. This is due to the increasing complexity of technical products, the need to improve efficiency, quality, reliability and durability.

It is clear that the requirements for strength and reliability of the structure are of paramount importance.

For practical work, the engineer needs to develop skills to create simple and clear models of phenomena and real objects, discarding secondary factors.

If the technical requirements for the product indicate the probability of failure-free operation $\mathrm{P}=0.99$, this means that for the resource (time of operation) may fail (on average) one product made of steel.

When evaluating strength reliability, the probability of failure is often determined 


$$
F=1-P
$$

Finding the probability of failure or failure - free operation at the product design stage is a very difficult task. Currently, the main method for evaluating strength reliability is to determine the strength reserves.

It is essential that the reliability and reliability of various structures, including the profile of the laser saw, is associated with the definition of the service life. In most cases, the service life refers to the time when the product is operating under load or load cycles [4]. New laser and Linter saws with a diameter of $320 \mathrm{~mm}$ have 280 and 330 teeth, respectively. Saws used in cotton mills that have teeth destruction in the form of abrasive wear, plastic crumpling and breakdowns are cut down to a smaller diameter in the saw repair shop.

The technical standard provides for 5 -fold cutting of teeth (Fig. 1) on saw disks with a decreasing diameter: $320,300,290,280,270$ and $260 \mathrm{~mm}$ [8]. After working off saws with a diameter of $260 \mathrm{~mm}$ on linters, they are not subject to restoration (renovation) and are passed for scrap.

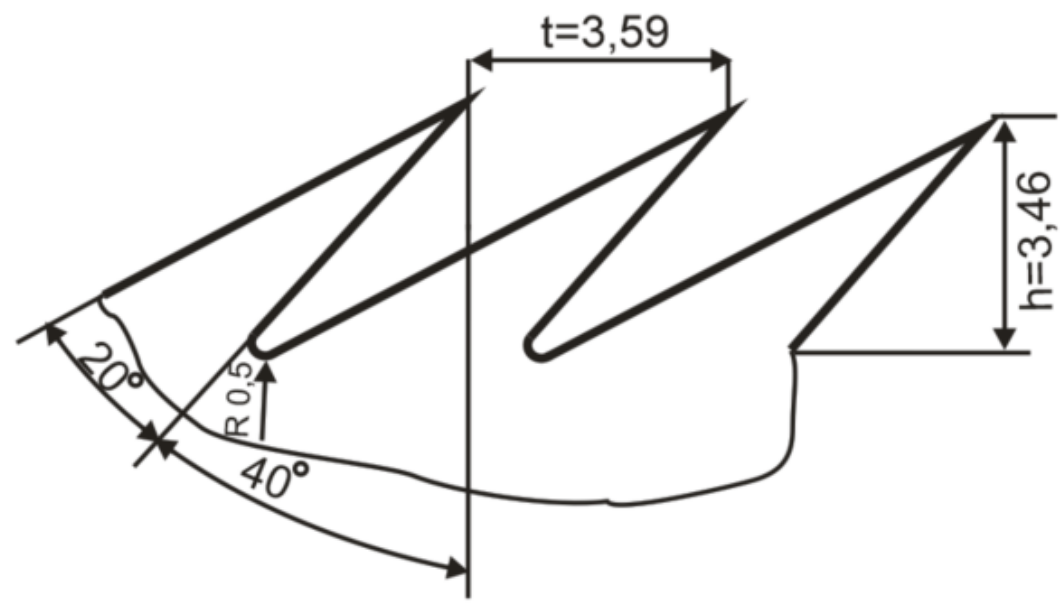

a)

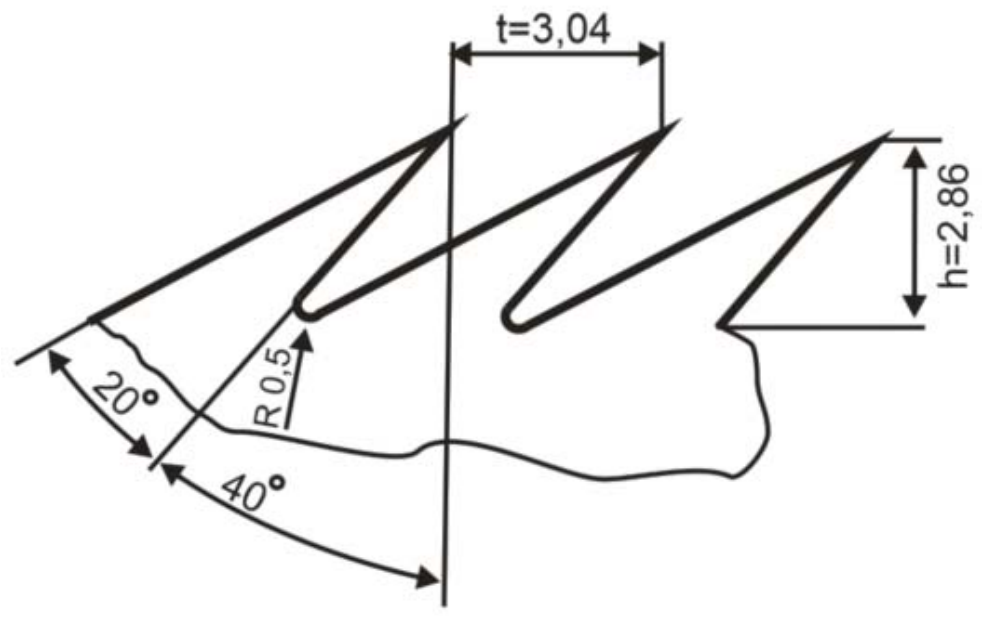

b)
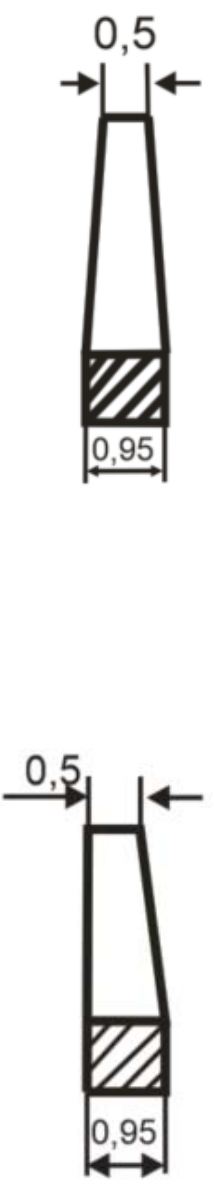

Fig. 1. Geometric parameters of saw blade teeth for gins (a) and linters (b)

The short inter-repair service life of saws (according to the technical regulations, chain and Linter saws are subject to replacement after 96 and 48 hours, respectively) requires frequent cutting operations for a smaller diameter, which increases their annual demand, which is more than one million pieces for the cotton gin industry of Uzbekistan.

\section{Model Specification And Parameter Estimation In FiXed Effect Model}

All paragraphs must be indented. All paragraphs must be justified, i.e. both left-justified and right-justified. 


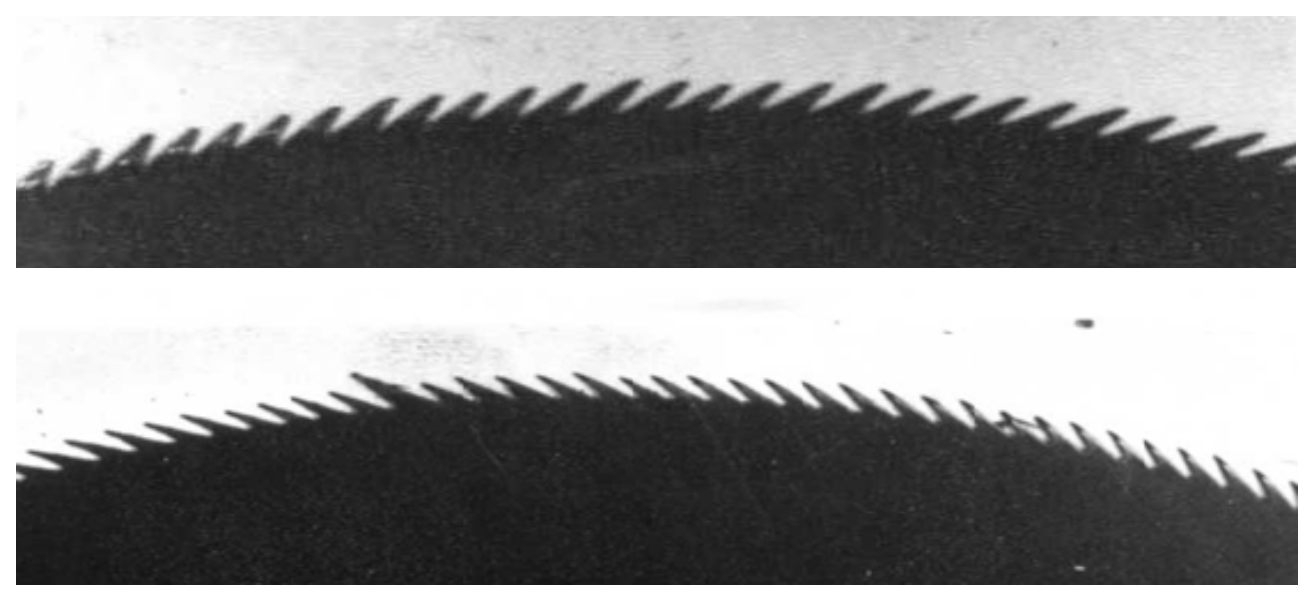

Fig. 2. Fragments of cut saw disk

Performance of gins and linters, their energy consumption, as well as quality of produced products (cotton fibre, lint, seeds) depend to a large extent on the efficiency of saw repair shops. Operations to prepare saw and saw cylinders in the saw repair shop of cotton plants include such activities as sorting, sharpening, cutting, rigging, sizing and grinding. In order to perform these operations, the saw repair shop must be equipped with tooth processing machines, saw points, machines for removing (grinding) chamfers from saw teeth [4]. It is necessary to have in sufficient quantity a cutting tool, a set of gauges and templates for quality control and saw geometry.
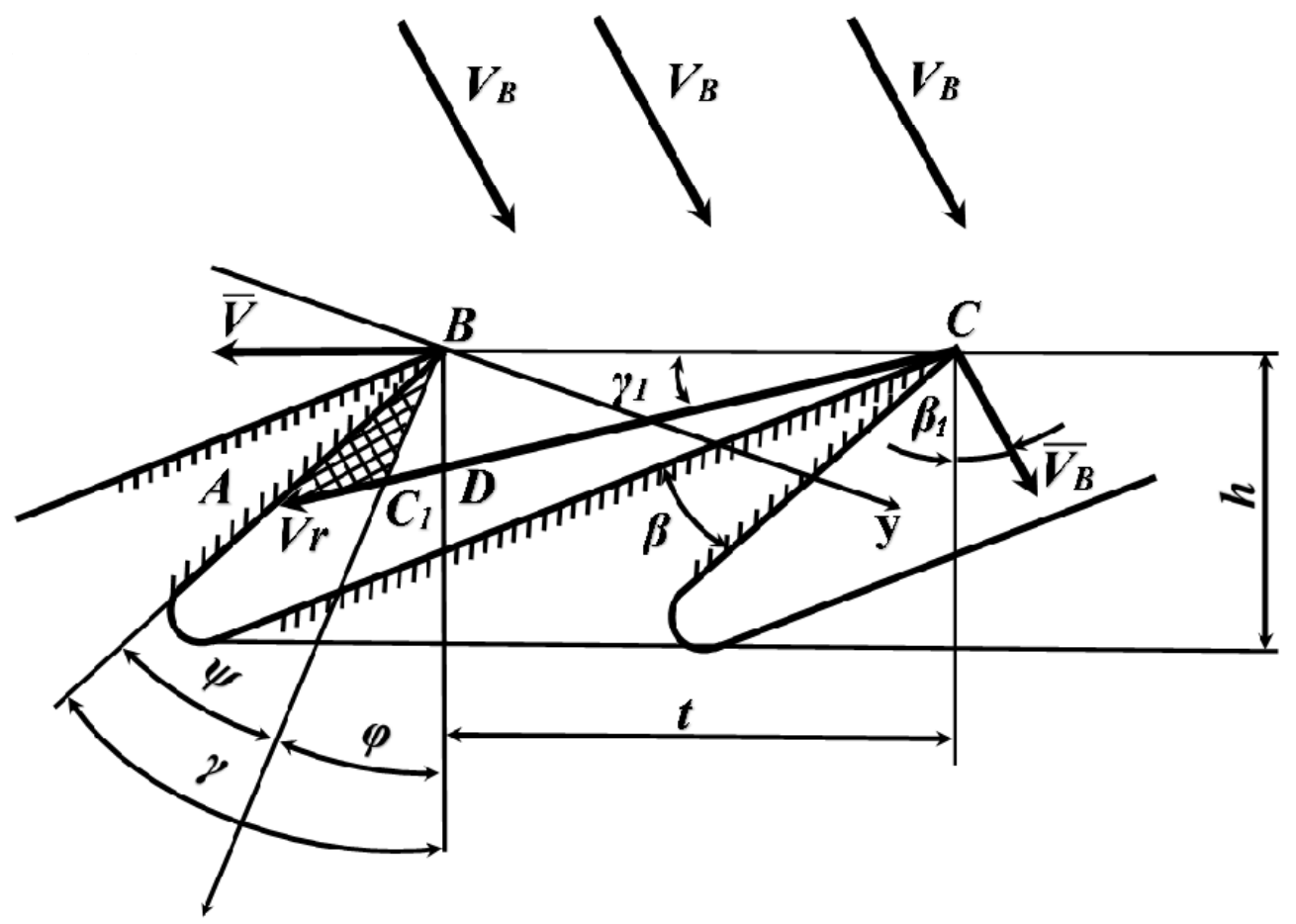

Fig 3. Force acting forces during ginning on working surfaces of teeth of existing

The fixed effect estimator allows $\alpha$ i to differ across units by estimating different constants for each crosssection. Standard errors are obtained from a re-estimated model with no intercept. We note that estimating the cross-section specific constant regression model with a large number of cross-section units is time consuming, and may result in estimates that are less accurate than those obtained using the fixed-effects option.

The influence of the inclination of the front face of the teeth for any pitch values of the teeth has little effect and is evident to the extent that this inclination affects the degree of dismembering of the fiber strands being torn off the seeds. However, due to the complexity of the processing, it is impractical to apply an inclination of the front face of the teeth of more than $40^{\circ}$, and an increase in the working space of the teeth significantly affects the quality and productivity of the gining. It follows from the above conclusions that the improvement of the profiles of the teeth of the gin saw in depth to study the force of the working part of the teeth acting in the 
process of operation and to justify the parameters, to recommend the optimal version of the profile of the gin saw.

Levkovich V. A. in these works for the first time theoretically justified the process of jining on saw gins and solved a number of theoretical and practical issues related to genie saw and construction of rational tooth profile [4]. The main findings of the studies were as follows:

1) In order to increase productivity and improve quality, it is necessary to:

Apply a straight-border tooth profile and a size corresponding to the Harvnietter gin tooth profile.

From this point on, the question of tooth shape was theoretically and practically considered correct, i.e. a tooth with straight faces is considered the most rational (Fig. 3.). But further development of science and practice confirmed the insufficiency of Levnikovich 's conclusion B. A.

2) The saw tooth grabs, cuts off the seeds and pulls the fibers behind the grate, and this alignment of the saw tooth operation phase affects the performance-the more the capture fibers and breaks the saw tooth off the seeds per unit time, the higher the performance of the gin.

3) The theoretical number of fibers captured by one genie saw tooth is:

$$
i=\frac{P_{z} \cdot 1000 \cdot p}{z \cdot n \cdot 60}
$$

From where:

$$
P_{z}=\frac{i \cdot n \cdot 60}{1000 \cdot p}
$$

Where: $\mathrm{P}_{\mathrm{z}}$ - saw capacity in $\mathrm{kg}$ fibre per hour;

$\mathrm{p}$-the amount of fibers in 1 gram;

$\mathrm{z}$-number of teeth on the saw;

$\mathrm{n}$-saw shaft RPM per minute;

i -number of fibers captured by one tooth.

After substitution comes the final formula of saw gin performance:

$$
P_{z}=k \cdot A \cdot s \cdot z \cdot n
$$

k-tooth cavity filling factor;

A-the result of all constant values;

s-area of the hollow of tooth.

On the basis of the above formula, the productivity is independent of the cotton grade and there is a function of tooth area, number of teeth and number of rotations of the saw roll (Fig. 4). Obviously, the value of the tooth cavity and the number of teeth per saw are linked to each other, it is necessary to find the optimal ratio of these values [5]. The tests carried out show that the best result of ginning by quantitative and qualitative indicators is obtained at the number of teeth in 280 per 12 saws.

4) Saw teeth are loaded with trapped fibers unevenly and not all of them participate in the work.

5) In order to better integrate the tooth with the cheese roll, which will contribute to an increase in fiber capture, and therefore productivity, the saw tooth needs to be made acutely. 

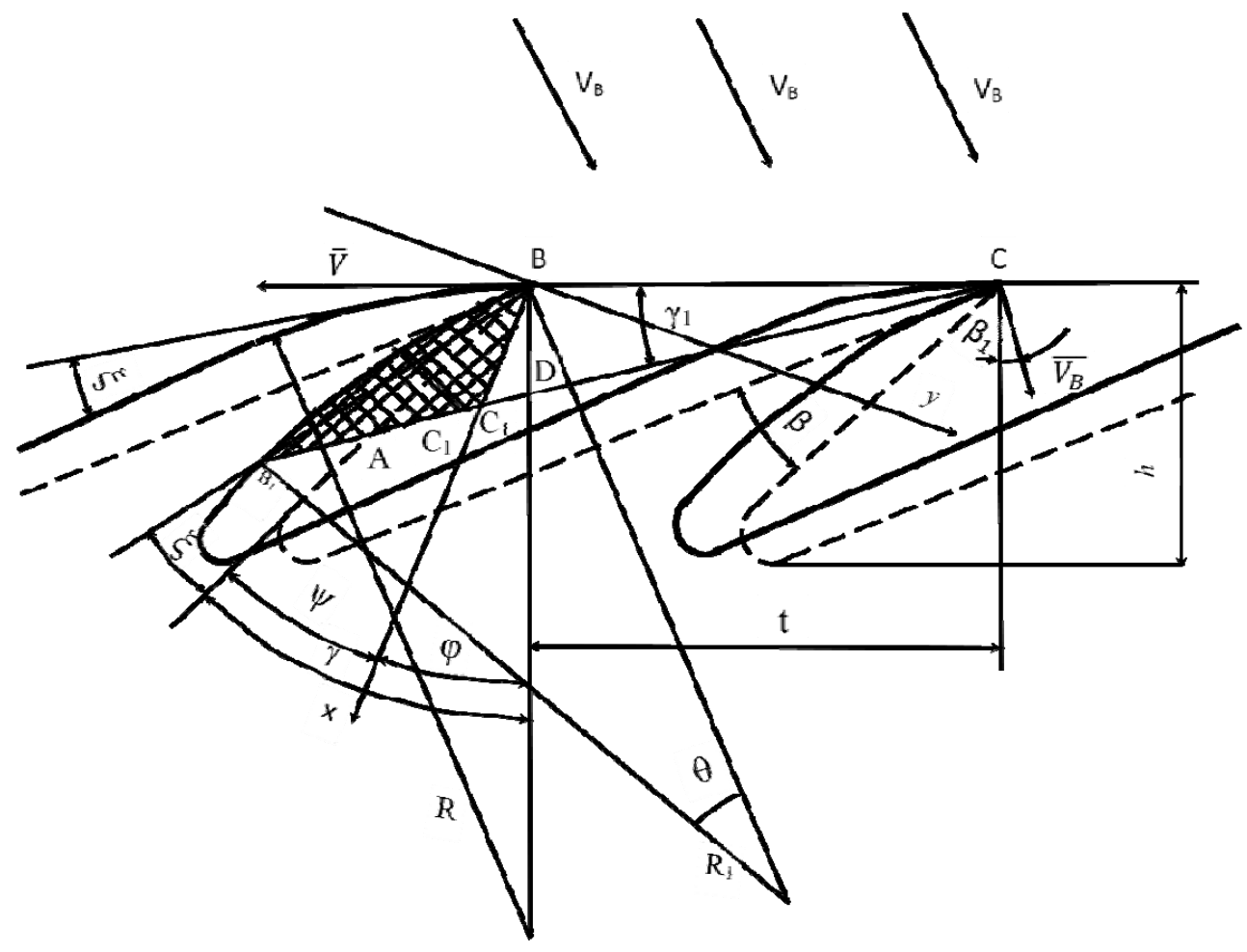

Rice 4. Influence of acting force in process of gingering on working sections of teeth.

On the basis of this model (Figure 4), which opens a significant reserve in the possibility of filling the teeth cavities with fibre, it is possible to use saws with reduced pitch of teeth (at their number on a saw more than 280). The use of such saws provides sufficient productivity while significantly reducing the interaction forces, and hence improving fibre quality and reducing seed damage.

\section{CONCLUSION}

The version of this template is V5. Most of the formatting instructions in this document have been compiled by Causal Productions from the IEEE LaTeX style files. Causal Productions offers both A4 templates and US Letter templates for LaTeX and Microsoft Word. The LaTeX templates depend on the official IEEEtran.cls and IEEEtran.bst files, whereas the Microsoft Word templates are self-contained. Causal Productions has used its best efforts to ensure that the templates have the same appearance.

- In all the theoretical analyses carried out, in which the saws during bench tests showed increased gripping capacity, and on natural gins - marginal productivity, at the same time there was also a decrease in fibre quality. The high gripping capacity of the teeth and saw in general is accompanied by increased force on the processing product, which causes a decrease in fibre quality and damage to seeds.

- Saw with diluted teeth was characterized by the highest performance indicators, and from the point of view of fibre quality these saws showed the worst result. The work of such saws caused increased powder formation and, above all, defects such as fiber skin and mechanical damage to seeds.

- Saws with increased pitch of teeth, i.e. with reduced number (less than 280), as well as saws with diluted teeth, provided increased efficiency and reduced fibre quality. However, the decrease in fiber and seed quality was less noticeable.

- Saws with reduced pitch of teeth, i.e. with increased number (more than 280), provided efficiency of ginning process, meeting requirements of modern cotton cleaning production, with marked improvement of quality of produced fibre and seeds. This can be explained by the fact that the potential ability to capture tooth cavity fibers, even with small dimensions, is sufficiently high to provide a gingering with high productivity.

\section{REFERENCES}

[1] V. S. Fyodorov, "Raw Cotton Primary Processing Technology", Gylenprom. 1937.

[2] B. A. Levkovich, "Elements of Jing Theory". State publishing house Ouse SSR. Tashkent, 1938.

[3] X. Saidov, On the rational profile of the tooth of the genie saw, Journal New Technology. GITH UzSSR by coordination. N / п works No. 3. 1962 of P. 22-24.

[4] B. A. Levkovich, "Rational tooth profile of fibre-separating saw". TTI Collection, No. 12, 1961

[5] S. Akhmedova, "On the tooth profile of the fibre-separating and puffing saw". TTI Collection, No. 6, 1958. 